\title{
Review of: "MetaCoAG: Binning Metagenomic Contigs via Composition, Coverage and Assembly Graphs"
}

\author{
Simon Rasmussen ${ }^{1}$ \\ 1 Copenhagen University
}

Potential competing interests: NB: I am a co-author of the VAMB paper

Very interesting and high quality work. I have two main questions:

1. As I understand it F1-score is reported for all bins returned by the individual methods and this could be an unfair comparison if they have different threshold of smallest bins that they report. E.g. a method reporting all bins compared to a method only reporting bins larger than for instance $500 \mathrm{~kb}$ is not a valid comparison in terms of F1. Please use a similar threshold for all bins. Furthermore, in principle users of the program will only be interested in bins larger than a certain size and/or that are deemed high quality using an evaluation program (for instance CheckM).

2. It is great to see that authors provide results using both co-assembly and single-sample assembly. I also commend that the authors provided the command lines used to run all binners. I noticed that VAMB was run without the multi-split option which is the preferred method and reported in that paper. It would be very interesting to see how it compares to this approach - just using the numbers from the VAMB paper it seems to produce more bins than any of the binners used (including MetaCoAG)? For instance, VAMB on CAMI2 Airways in the VAMB manuscript is $60 \mathrm{HQ}$ bins compared to only 20 reported in Supplementary Table 6 of this paper (where MetaCoAG produce 33 HQ bins).

NB: I am a co-author of the VAMB paper 